\title{
Feedback Control Variables Have No Influence on the Permanence of a Discrete $N$-Species Cooperation System
}

\author{
Liujuan Chen, ${ }^{1}$ Xiangdong $\mathrm{Xie}_{,}{ }^{2}$ and Lijuan Chen ${ }^{3}$ \\ ${ }^{1}$ Ministry of Science Training, Fujian Institute of Education, Fuzhou, Fujian 350001, China \\ ${ }^{2}$ Department of Mathematics, Ningde Teachers College, Ningde, Fujian 352100, China \\ ${ }^{3}$ College of Mathematics and Computer Science, Fuzhou University, Fuzhou, Fujian 350002, China
}

Correspondence should be addressed to Liujuan Chen, clj1018@sohu.com

Received 14 March 2009; Revised 28 June 2009; Accepted 27 July 2009

Recommended by Leonid Berezansky

A new set of sufficient conditions for the permanence of a discrete $N$-species cooperation system with delays and feedback controls are obtained. Our result shows that feedback control variables have no influence on the persistent property of the discrete cooperative system, thus improves and supplements the main result of F. D. Chen (2007).

Copyright (C) 2009 Liujuan Chen et al. This is an open access article distributed under the Creative Commons Attribution License, which permits unrestricted use, distribution, and reproduction in any medium, provided the original work is properly cited.

\section{Introduction}

The aim of this paper is to investigate the permanent property of the following nonautonomous discrete $n$-species cooperation system with time delays and feedback controls of the form:

$$
\begin{gathered}
x_{i}(k+1)=x_{i}(k) \exp \left\{r_{i}(k)\left[1-\frac{x_{i}\left(k-\tau_{i i}\right)}{a_{i}(k)+\sum_{j=1, j \neq i}^{n} b_{i j}(k) x_{j}\left(k-\tau_{i j}\right)}-c_{i}(k) x_{i}\left(k-\tau_{i i}\right)\right]\right. \\
\left.-d_{i}(k) u_{i}(k)-e_{i}(k) u_{i}\left(k-\eta_{i}\right)\right\}, \\
\Delta u_{i}(k)=-\alpha_{i}(k) u_{i}(k)+\beta_{i}(k) x_{i}(k)+\gamma_{i}(k) x_{i}\left(k-\sigma_{i}\right),
\end{gathered}
$$

where $x_{i}(k)(i=1, \ldots, n)$ is the density of cooperation species $x_{i}, u_{i}(k)(i=1, \ldots, n)$ is the control variable (see $[1,2])$. 
Throughout this paper, we assume the following.

$\left(H_{1}\right) r_{i}(k), a_{i}(k), b_{i j}(k), c_{i}(k), d_{i}(k), e_{i}(k), \alpha_{i}(k), \beta_{i}(k), \gamma_{i}(k), i, j=1,2, \ldots, n$ are all bounded nonnegative sequences such that

$$
\begin{array}{cc}
0<r_{i}^{l} \leq r_{i}^{u}, \quad 0<a_{i}^{l} \leq a_{i}^{u}, \quad 0<b_{i j}^{l} \leq b_{i j}^{u}, \\
0<c_{i}^{l} \leq c_{i}^{u}, \quad 0<d_{i}^{l} \leq d_{i}^{u}, \quad 0 \leq e_{i}^{l} \leq e_{i}^{u}, \\
0<\alpha_{i}^{l} \leq \alpha_{i}^{u}<1, \quad 0<\beta_{i}^{l} \leq \beta_{i}^{u}, \quad 0<\gamma_{i}^{l} \leq \gamma_{i}^{u} .
\end{array}
$$

Here, for any bounded sequence $\{h(k)\}$ and $N=\{0,1,2, \ldots\}, h^{u}=\sup _{k \in N}\{h(k)\}$ and $h^{l}=$ $\inf _{k \in N}\{h(k)\}$.

$\left(H_{2}\right) \tau_{i j}, \eta_{i}, \sigma_{i}, i, j=1,2, \ldots, n$ are all nonnegative integers.

Let $\tau=\max \left\{\tau_{i j}, \sigma_{i}, \eta_{i}, i, j=1,2, \ldots, n\right\}$; we consider (1.1) together with the following initial conditions:

$$
\begin{aligned}
& x_{i}(\theta)=\varphi_{i}(\theta) \geq 0, \quad \theta \in N[-\tau, 0]=\{-\tau,-\tau+1, \ldots, 0\}, \varphi_{i}(0)>0, \\
& u_{i}(\theta)=\psi_{i}(\theta) \geq 0, \quad \theta \in N[-\tau, 0]=\{-\tau,-\tau+1, \ldots, 0\}, \psi_{i}(0)>0 .
\end{aligned}
$$

It is not difficult to see that the solutions of (1.1)-(1.3) are well defined for all $k \geq 0$ and satisfy

$$
x_{i}(k)>0, \quad u_{i}(k)>0, \quad \text { for } k \in Z, i=1,2, \ldots, n,
$$

where $Z$ is the set of integer numbers.

Recently, Chen [3] proposed and studied the permanence of system (1.1). Set

$$
M_{i 1}=\frac{\exp \left\{r_{i}^{u}\left(\tau_{i i}+1\right)-1\right\}}{c_{i}^{l} r_{i}^{u}}, \quad M_{i 2}=\frac{\left(\beta_{i}^{u}+\gamma_{i}^{u}\right) M_{i 1}}{\alpha_{i}^{l}}
$$

Using the comparison theorem, he obtained the following result.

Theorem A (see [3]). Assume that $\left(H_{1}\right)$ and $\left(H_{2}\right)$ hold, and assume further that $\left(H_{3}\right)$

$$
r_{i}^{l}>\left(d_{i}^{u}+e_{i}^{u}\right) M_{i 2}, \quad i=1,2, \ldots, n
$$

holds, then system (1.1) is permanent.

However, as was pointed out by Fan and Wang [4], "if we use the method of comparison theorem, then the additional condition, in some extent, is necessary. But for the system itself, this condition may not necessary." In [4], by establishing a new difference inequality, Fan and Wang showed that feedback control has no influence on the permanence of a single species discrete model. Their success motivated us to consider the persistent property of system (1.1). Indeed, in this paper, we will develop the analysis idea of [3] and apply the difference inequality obtained by Fan and Wang [4] to prove the following result. 
Theorem 1.1. Assume that $\left(H_{1}\right)$ and $\left(H_{2}\right)$ hold, then system (1.1) is permanent.

Remark 1.2. Theorem 1.1 shows that feedback control variables have no influence on the permanent property of system (1.1). It is natural to ask whether the feedback control variables have the influence on the stability property of the system or not. At present, we had difficulty to give an affirm answer to this problem, and we will leave this in our future study.

We will prove Theorem 1.1 in the next section. For more works on cooperative system and feedback control ecosystem, one could refer to [1-23] and the references cited therein.

\section{Proof of Theorem 1.1}

Now we state several lemmas which will be useful for the proof of our main result.

Lemma 2.1 (see [5, page 125]). Consider the first-order difference equation

$$
y(k+1)=A y(k)+B, \quad k=1,2, \ldots,
$$

where $A$ and $B$ are positive constants. Assume that $|A|<1$, for any initial value $y(0)$, there exist a unique solution $y(k)$ of (2.1) which can be expressed as follows: $y(k)=A^{k}\left(y(0)-y^{*}\right)+y^{*}$, where $y^{*}=B /(1-A)$. Thus, for any solution $\{y(k)\}$ of system (2.1), one has

$$
\lim _{k \rightarrow+\infty} y(k)=y^{*}
$$

Lemma 2.2 (see [5, page 241] (Comparison theorem)). Let $k \in N_{k_{0}}^{+}=\left\{k_{0}, k_{0}+1, \ldots, k_{0}+\right.$ $l, \ldots\}, r \geq 0$. For any fixed $k, g(k, r)$ is a nondecreasing function, and for $k \geq k_{0}$, the following inequalities hold:

$$
\begin{aligned}
& y(k+1) \leq g(k, y(k)), \\
& u(k+1) \geq g(k, u(k)) .
\end{aligned}
$$

If $y\left(k_{0}\right) \leq u\left(k_{0}\right)$, then $y(k) \leq u(k)$ for all $k \geq k_{0}$.

Lemma 2.3 (see [6, Theorem 2.1]). Consider the following single species discrete model:

$$
N(k+1)=N(k) \exp \left(r(k)\left(1-\frac{N(k)}{h(k)}\right)\right)
$$

where $\{r(k)\}$ and $\{h(k)\}$ are strictly positive sequences of real numbers defined for $k \in N=$ $\{0,1,2, \ldots\}$ and $0<h^{l} \leq h^{u}, 0<r^{l} \leq r^{u}$. Any solution of system (2.4) with initial condition $N(0)>$ 0 satisfies $m \leq \liminf _{k \rightarrow+\infty} N(k) \leq \limsup _{k \rightarrow+\infty} N(k) \leq M$, where $M=\left(h^{u} / r^{u}\right) \exp \left(r^{u}-\right.$ 1), $m=h^{l} \exp \left(r^{u}\left(1-M / h^{l}\right)\right)$.

Lemma 2.4 (see [7]). Assume that $\{x(k)\}$ satisfies

$$
x(k+1) \geq x(k) \exp \{a(k)-b(k) x(k)\}, \quad k \geq N_{0},
$$


$\lim \sup _{k \rightarrow+\infty} x(k) \leq x^{*}$, and $x\left(N_{0}\right)>0$, where $a(k)$ and $b(k)$ are nonnegative sequences bounded above and below by positive constants and $N_{0} \in N$. Then

$$
\liminf _{k \rightarrow+\infty} x(k) \geq \min \left\{\frac{a^{l}}{b^{u}} \exp \left\{a^{l}-b^{u} x^{*}\right\}, \frac{a^{l}}{b^{u}}\right\}
$$

Lemma 2.5 (see $[4])$. Assume that $A>0$ and $y(0)>0$. Further suppose that

(i)

$$
y(n+1) \leq A y(n)+B(n), \quad n=1,2, \ldots
$$

then for any integer $k \leq n, y(n) \leq A^{k} y(n-k)+\sum_{i=0}^{k-1} A^{i} B(n-i-1)$. Especially, if $A<1$ and $B$ is bounded above with respect to $M$, then $\lim _{\sup _{t \rightarrow+\infty}} y(n) \leq M /(1-A)$;

(ii)

$$
y(n+1) \geq A y(n)+B(n), \quad n=1,2, \ldots,
$$

then for any integer $k \leq n, y(n) \geq A^{k} y(n-k)+\sum_{i=0}^{k-1} A^{i} B(n-i-1)$. Especially, if $A<1$ and $B$ is bounded below with respect to $m^{*}$, then $\liminf _{t \rightarrow+\infty} y(n) \geq m^{*} /(1-A)$.

Lemma 2.6. Let $(x(k), u(k))^{T}=\left(x_{1}(k), \ldots, x_{n}(k), u_{1}(k), \ldots, u_{n}(k)\right)^{T}$ be any positive solution of system (1.1), there exists a positive constant $M$, which is independent of the solution of system (1.1), such that

$$
\limsup _{k \rightarrow+\infty} x_{i}(k) \leq M ; \quad \limsup _{k \rightarrow+\infty} u_{i}(k) \leq M, \quad i=1,2, \ldots, n .
$$

Proof. Let $(x(k), u(k))^{T}=\left(x_{1}(k), \ldots, x_{n}(k), u_{1}(k), \ldots, u_{n}(k)\right)^{T}$ be any positive solution of system (1.1); similarly to the proof of Theorem 2.1 in [3], we have

$$
\limsup _{k \rightarrow+\infty} x_{i}(k) \leq M_{i 1}, \quad \limsup _{k \rightarrow+\infty} u_{i}(k) \leq M_{i 2}
$$

where $M_{i 1}, M_{i 2}, i=1,2, \ldots, n$ are defined by (1.5). In fact, from the $i$ th equation of (1.1), it follows that

$$
x_{i}(k+1) \leq x_{i}(k) \exp \left\{r_{i}(k)\right\}
$$

Let $x_{i}(k)=\exp \left\{N_{i}(k)\right\}$, then (2.11) is equivalent to

$$
N_{i}(k+1)-N_{i}(k) \leq r_{i}(k) .
$$


Summing both sides of (2.12) from $k-\tau_{i i}$ to $k-1$ leads to

$$
\sum_{j=k-\tau_{i i}}^{k-1}\left(N_{i}(j+1)-N_{i}(j)\right) \leq \sum_{j=k-\tau_{i i}}^{k-1} r_{i}(j) \leq r_{i}^{u} \tau_{i i}
$$

We obtain that $N_{i}\left(k-\tau_{i i}\right) \geq N_{i}(k)-r_{i}^{u} \tau_{i i}$ and hence,

$$
x_{i}\left(k-\tau_{i i}\right) \geq x_{i}(k) \exp \left\{-r_{i}^{u} \tau_{i i}\right\}
$$

Substituting (2.14) to the $i$ th equation of (1.1), it immediately follows that

$$
\begin{aligned}
x_{i}(k+1) & \leq x_{i}(k) \exp \left[r_{i}(k)\left(1-c_{i}(k) x_{i}\left(k-\tau_{i i}\right)\right)\right] \\
& \leq x_{i}(k) \exp \left[r_{i}(k)\left(1-c_{i}(k) x_{i}(k) \exp \left\{-r_{i}^{u} \tau_{i i}\right\}\right)\right] .
\end{aligned}
$$

By applying Lemmas 2.2 and 2.3 to (2.15), we have

$$
\limsup _{k \rightarrow+\infty} x_{i}(k) \leq \frac{\exp \left\{r_{i}^{u}\left(\tau_{i i}+1\right)-1\right\}}{c_{i}^{l} r_{i}^{u}}=M_{i 1}
$$

For any small enough $\epsilon>0$, it follows from (2.16) that there exists enough large $K_{1}$ such that

$$
x_{i}(k) \leq M_{i 1}+\epsilon, \quad \text { for } k \geq K_{1} .
$$

This, together with $(n+i)$ th equation of $(1.1)$, leads to

$$
\Delta u_{i}(k) \leq-\alpha_{i}(k) u_{i}(k)+\left(\beta_{i}(k)+\gamma_{i}(k)\right)\left(M_{i 1}+\epsilon\right), \quad \text { for } k \geq K_{1}+\tau
$$

And so,

$$
u_{i}(k+1) \leq\left(1-\alpha_{i}^{l}\right) u_{i}(k)+\left(\beta_{i}^{u}+\gamma_{i}^{u}\right)\left(M_{i 1}+\epsilon\right), \quad \text { for } k \geq K_{1}+\tau
$$

Notice that $0<1-\alpha_{i}^{l}<1$; it follows from (2.19) and Lemmas 2.1 and 2.2 that $\lim \sup _{k \rightarrow+\infty} u_{i}(k) \leq\left(\beta_{i}^{u}+\gamma_{i}^{u}\right)\left(M_{i 1}+\epsilon\right) / \alpha_{i}^{l}$. Let $\epsilon \rightarrow 0$ in above inequality, then

$$
\limsup _{k \rightarrow+\infty} u_{i}(k) \leq \frac{\left(\beta_{i}^{u}+\gamma_{i}^{u}\right) M_{i 1}}{\alpha_{i}^{l}}=M_{i 2}
$$

Set $M=\max _{i}\left\{M_{i 1}, M_{i 2}\right\}$. The conclusion of Lemma 2.6 holds. The proof is complete. 
Lemma 2.7. Let $(x(k), u(k))^{T}=\left(x_{1}(k), \ldots, x_{n}(k), u_{1}(k), \ldots, u_{n}(k)\right)^{T}$ be any positive solution of system (1.1), there exists a positive constant $m$, which is independent of the solution of system (1.1), such that

$$
\liminf _{k \rightarrow+\infty} x_{i}(k) \geq m ; \quad \liminf _{k \rightarrow+\infty} u_{i}(k) \geq m
$$

Proof. Let $(x(k), u(k))^{T}=\left(x_{1}(k), \ldots, x_{n}(k), u_{1}(k), \ldots, u_{n}(k)\right)^{T}$ be a solution of system (1.1) satisfying the initial condition (1.3). From Lemma 2.6, there exists a $K_{1}$ such that for all $k \geq$ $K_{1}, x_{i}(t) \leq 2 M_{i 1}, u_{i}(k) \leq 2 M_{i 2}$. Thus, for $k>K_{1}+\tau$, from the $i$ th equation of system (1.1), it follows that

$$
\begin{aligned}
x_{i}(k+1) & \geq x_{i}(k) \exp \left\{r_{i}(k)\left(1-\frac{x_{i}\left(k-\tau_{i i}\right)}{a_{i}^{l}}-c_{i}^{u} x_{i}\left(k-\tau_{i i}\right)\right)-2\left(d_{i}^{u}+e_{i}^{u}\right) M_{i 2}\right\} \\
& \geq x_{i}(k) \exp \left\{r_{i}^{l}\left(1-\frac{2 M_{i 1}}{a_{i}^{l}}-2 c_{i}^{u} M_{i 1}\right)-2\left(d_{i}^{u}+e_{i}^{u}\right) M_{i 2}\right\} \\
& \geq x_{i}(k) \exp \left\{-\frac{2 r_{i}^{l} M_{i 1}}{a_{i}^{l}}-2 r_{i}^{l} c_{i}^{u} M_{i 1}-2\left(d_{i}^{u}+e_{i}^{u}\right) M_{i 2}\right\} \\
& \stackrel{\text { def }}{=} x_{i}(k) \exp \left\{\zeta_{i}\right\} .
\end{aligned}
$$

Obviously, $\zeta_{i}$ is a negative constant. Let $x_{i}(k)=\exp \left\{N_{i}(k)\right\}$, the above inequality is equivalent to

$$
N_{i}(k+1)-N_{i}(k) \geq \zeta_{i}
$$

Summing both sides of (2.23) from $k-m$ to $k-1$ leads to $\sum_{j=k-m}^{k-1}\left(N_{i}(j+1)-N_{i}(j)\right) \geq \zeta_{i} m$, and so, $N_{i}(k-m) \leq N_{i}(k)-\zeta_{i} m$, therefore,

$$
x_{i}(k-m) \leq x_{i}(k) \exp \left\{-\zeta_{i} m\right\} .
$$

Specially, we have

$$
\begin{aligned}
& x_{i}\left(k-\sigma_{i}\right) \leq x_{i}(k) \exp \left\{-\zeta_{i} \sigma_{i}\right\} \leq x_{i}(k) \exp \left\{-\zeta_{i} \tau\right\}, \\
& x_{i}\left(k-\tau_{i i}\right) \leq x_{i}(k) \exp \left\{-\zeta_{i} \tau_{i i}\right\} \leq x_{i}(k) \exp \left\{-\zeta_{i} \tau\right\}, \\
& x_{i}\left(k-\eta_{i}\right) \leq x_{i}(k) \exp \left\{-\zeta_{i} \eta_{i}\right\} \leq x_{i}(k) \exp \left\{-\zeta_{i} \tau\right\} .
\end{aligned}
$$


Substituting the first inequality into the $(n+i)$ th equation of system (1.1) leads to

$$
\begin{aligned}
u_{i}(k+1) & \leq\left(1-\alpha_{i}(k)\right) u_{i}(k)+\beta_{i}(k) x_{i}(k)+\gamma_{i}(k) x_{i}(k) \exp \left\{-\zeta_{i} \tau\right\} \\
& \leq\left(1-\alpha_{i}^{l}\right) u_{i}(k)+\beta_{i}^{u} x_{i}(k)+\gamma_{i}^{u} x_{i}(k) \exp \left\{-\zeta_{i} \tau\right\}=A_{i} u_{i}(k)+B_{i} x_{i}(k),
\end{aligned}
$$

where $A_{i}=1-\alpha_{i}^{l}, B_{i}=\beta_{i}^{u}+\gamma_{i}^{u} \exp \left\{-\zeta_{i} \tau\right\}$. Then Lemma 2.5 and (2.24) imply that, for any integer $s \leq k$,

$$
\begin{aligned}
u_{i}(k) & \leq A_{i}^{s} u_{i}(k-s)+\sum_{j=0}^{s-1} B_{i} x_{i}(k-j-1) \\
& \leq A_{i}^{s} u_{i}(k-s)+\sum_{j=0}^{s-1} B_{i} \exp \left\{-\zeta_{i}(j+1)\right\} x_{i}(k) .
\end{aligned}
$$

Note that $0<1-\alpha_{i}^{l}<1$ and for enough large $k$, s, which satisfy $k-s \geq K_{1}$, then $u_{i}(k-s) \leq 2 M$ and $\lim _{s \rightarrow+\infty} A_{i}^{s}=0$. Thus, for $k, s \rightarrow+\infty$ and $k-s \geq K_{1}, 0 \leq A_{i}^{s} u_{i}(k-s) \leq 2 A_{i}^{s} M \rightarrow 0$. Then, there exists a positive integer $K_{2}>K_{1}$ such that for any positive solution of system (1.1), $2\left(d_{i}^{u}+e_{i}^{u}\right) A_{i}^{s} M \leq(1 / 2) r_{i}^{l}$, for all $s \geq K_{2}$ and $i=1,2, \ldots, n$. In fact, we could choose $K_{2}=$ $\max _{i}\left\{\left|\ln C_{i} / \ln A_{i}\right|\right\}$, where $C_{i}=(1 / 2) r_{i}^{l} / 2 M\left(d_{i}^{u}+e_{i}^{u}\right), i=1,2, \ldots, n$. Fix $K_{2}$, for $k>K_{2}+K_{1}$, we get

$$
\begin{aligned}
u_{i}(k) & \leq A_{i}^{K_{2}} u_{i}\left(k-K_{2}\right)+\sum_{j=0}^{K_{2}-1} B_{i} x_{i}(k-j-1) \\
& \leq 2 A_{i}^{K_{2}} M+\sum_{j=0}^{K_{2}-1} B_{i} \exp \left\{-\zeta_{i}(j+1)\right\} x_{i}(k) \\
& \stackrel{\text { def }}{=} 2 A_{i}^{K_{2}} M+D_{i} x_{i}(k) .
\end{aligned}
$$

And so, for $k>K_{2}+K_{1}+\tau$, we have

$$
u_{i}\left(k-\eta_{i}\right) \leq 2 A_{i}^{K_{2}} M+D_{i} x_{i}\left(k-\eta_{i}\right) .
$$


Substituting (2.28) and (2.29) into the ith equation of system (1.1), this together with (2.25) leads to (note that $\left.2\left(d_{i}^{u}+e_{i}^{u}\right) A_{i}^{K_{2}} M \leq(1 / 2) r_{i}^{l}\right)$

$$
\begin{aligned}
& x_{i}(k+1) \geq x_{i}(k) \exp \left[r_{i}(k)\left(1-\left(\frac{1}{a_{i}^{l}}+c_{i}^{u}\right) \exp \left\{-\zeta_{i} \tau\right\} x_{i}(k)\right)\right. \\
& \left.-d_{i}(k)\left(2 A_{i}^{K_{2}} M+D_{i} x_{i}(k)\right)-e_{i}(k)\left(2 A_{i}^{K_{2}} M+D_{i} x_{i}\left(k-\eta_{i}\right)\right)\right] \\
& \geq x_{i}(k) \exp \left[r_{i}(k)\left(1-\left(\frac{1}{a_{i}^{l}}+c_{i}^{u}\right) \exp \left\{-\zeta_{i} \tau\right\} x_{i}(k)\right)\right. \\
& \left.-d_{i}(k)\left(2 A_{i}^{K_{2}} M+D_{i} x_{i}(k)\right)-e_{i}(k)\left(2 A_{i}^{K_{2}} M+D_{i} \exp \left\{-\zeta_{i} \tau\right\} x_{i}(k)\right)\right] \\
& =x_{i}(k) \exp \left[\left(r_{i}(k)-2\left(d_{i}(k)+e_{i}(k)\right) 2 A_{i}^{K_{2}} M\right)\right. \\
& \left.-\left(r_{i}(k)\left(\frac{1}{a_{i}^{l}}+c_{i}^{u}\right) \exp \left\{-\zeta_{i} \tau\right\}+d_{i}(k) D_{i}+e_{i}(k) D_{i} \exp \left\{-\zeta_{i} \tau\right\}\right) x_{i}(k)\right] \\
& \geq x_{i}(k) \exp \left[\left(r_{i}^{l}-2\left(d_{i}^{u}+e_{i}^{u}\right) 2 A_{i}^{K_{2}} M\right)\right. \\
& \left.-\left(r_{i}^{u}\left(\frac{1}{a_{i}^{l}}+c_{i}^{u}\right) \exp \left\{-\zeta_{i} \tau\right\}+d_{i}^{u} D_{i}+e_{i}^{u} D_{i} \exp \left\{-\zeta_{i} \tau\right\}\right) x_{i}(\mathrm{k})\right] \\
& \geq x_{i}(k) \exp \left[\frac{1}{2} r_{i}^{l}-E_{i} x_{i}(k)\right] \text {, }
\end{aligned}
$$

where $E_{i}=r_{i}^{u}\left(1 / a_{i}^{l}+c_{i}^{u}\right) \exp \left\{-\zeta_{i} \tau\right\}+d_{i}^{u} D_{i}+e_{i}^{u} D_{i} \exp \left\{-\zeta_{i} \tau\right\}$

By applying Lemma 2.4 to (2.30), it immediately follows that

$$
\liminf _{k \rightarrow+\infty} x_{i}(k) \geq m_{i 1}
$$

where $m_{i 1}=\min \left\{(1 / 2) r_{i}^{l} / E_{i},\left((1 / 2) r_{i}^{l} / E_{i}\right) \exp \left\{(1 / 2) r_{i}^{l}-E_{i} M\right\}\right\}$.

From (2.31), we know that there exists enough large $K_{3}>K_{2}+K_{1}+\tau$ such that

$$
x_{i}(k) \geq \frac{1}{2} m_{i 1}, \quad \text { for } k \geq K_{3}+\tau \text {. }
$$

This together with the $(n+i)$ th equation of (1.1) leads to

$$
\Delta u_{i}(k) \geq-\alpha_{i}(k) u_{i}(k)+\frac{1}{2}\left(\beta_{i}(k)+\gamma_{i}(k)\right) m_{i 1}, \quad \text { for } k \geq K_{3}+\tau .
$$


And so,

$$
u_{i}(k+1) \geq\left(1-\alpha_{i}^{u}\right) u_{i}(k)+\frac{1}{2}\left(\beta_{i}^{l}+\gamma_{i}^{l}\right) m_{i 1}, \quad \text { for } k \geq K_{3}+\tau
$$

Noticing that $0<1-\alpha_{i}^{u}<1$ and applying Lemmas 2.1 and 2.2 to (2.34), we have

$$
\liminf _{k \rightarrow+\infty} u_{i}(k) \geq \frac{(1 / 2)\left(\beta_{i}^{l}+\gamma_{i}^{l}\right) m_{i 1}}{\alpha_{i}^{u}} \stackrel{\text { def }}{=} m_{i 2} .
$$

Setting $m=\min _{i}\left\{m_{i 1}, m_{i 2}\right\}$, the conclusion of Lemma 2.7 follows. This ends the proof of Lemma 2.7.

Proof of Theorem 1.1. Lemmas 2.6 and 2.7 show that under the assumptions $\left(H_{1}\right)$ and $\left(H_{2}\right)$, for any positive solution $(x(k), u(k))=\left(x_{1}(k), \ldots, x_{n}(k), u_{1}(k), \ldots, u_{n}(k)\right)^{T}$ of system $(1.1)$, one has

$$
\begin{aligned}
& m \leq \liminf _{k \rightarrow+\infty} x_{i}(k) \leq \limsup _{k \rightarrow+\infty} x_{i}(k) \leq M, \\
& m \leq \liminf _{k \rightarrow+\infty} u_{i}(k) \leq \limsup _{k \rightarrow+\infty} u_{i}(k) \leq M,
\end{aligned}
$$

where $m$ and $M$ are independent of the solution of system (1.1), thus, system (1.1) is permanent. This ends the proof of Theorem 1.1.

\section{Conclusions}

Stimulated by the works of Fan and Wang [4], in this paper, we revisit the model proposed by Chen [3]. We showed that condition $\left(\mathrm{H}_{3}\right)$ in [3] is not necessary to ensure the permanence of the system, which means that feedback control variables have no influence on the persistent property of system (1.1).

\section{Acknowledgments}

The authors are grateful to anonymous referees for their excellent suggestions, which greatly improved the presentation of the paper. Also, this work was supported by the Foundation of Education Department of Fujian Province (JA08253).

\section{References}

[1] M. Fan, K. Wang, P. J. Y. Wong, and R. P. Agarwal, "Periodicity and stability in periodic $n$ species Lotka-Volterra competition system with feedback controls and deviating arguments," Acta Mathematica Sinica, vol. 19, no. 4, pp. 801-822, 2003.

[2] H.-F. Huo and W.-T. Li, "Positive periodic solutions of a class of delay differential system with feedback control," Applied Mathematics and Computation, vol. 148, no. 1, pp. 35-46, 2004.

[3] F. Chen, "Permanence of a discrete $N$-species cooperation system with time delays and feedback controls," Applied Mathematics and Computation, vol. 186, no. 1, pp. 23-29, 2007. 
[4] Y.-H. Fan and L.-L. Wang, "Permanence for a discrete model with feedback control and delay," Discrete Dynamics in Nature and Society, vol. 2008, Article ID 945109, 8 pages, 2008.

[5] L. Wang and M. Q. Wang, Ordinary Difference Equation, Xinjiang University Press, Xinjiang, China, 1991.

[6] Z. Zhou and X. Zou, "Stable periodic solutions in a discrete periodic logistic equation," Applied Mathematics Letters, vol. 16, no. 2, pp. 165-171, 2003.

[7] F. Chen, "Permanence for the discrete mutualism model with time delays," Mathematical and Computer Modelling, vol. 47, no. 3-4, pp. 431-435, 2008.

[8] K. Gopalsamy, Stability and Oscillations in Delay Differential Equations of Population Dynamics, vol. 74 of Mathematics and Its Applications, Kluwer Academic Publishers, Dordrecht, The Netherlands, 1992.

[9] F. Chen, X. Liao, and Z. Huang, "The dynamic behavior of $N$-species cooperation system with continuous time delays and feedback controls," Applied Mathematics and Computation, vol. 181, no. 2, pp. 803-815, 2006.

[10] P. Weng, "Existence and global stability of positive periodic solution of periodic integrodifferential systems with feedback controls," Computers $\mathcal{E}$ Mathematics with Applications, vol. 40, no. 6-7, pp. 747$759,2000$.

[11] Y. Xiao, S. Tang, and J. Chen, "Permanence and periodic solution in competitive system with feedback controls," Mathematical and Computer Modelling, vol. 27, no. 6, pp. 33-37, 1998.

[12] K. Gopalsamy and P. X. Weng, "Feedback regulation of logistic growth," International Journal of Mathematics and Mathematical Sciences, vol. 16, no. 1, pp. 177-192, 1993.

[13] F. Chen, "Global asymptotic stability in $n$-species non-autonomous Lotka-Volterra competitive systems with infinite delays and feedback control," Applied Mathematics and Computation, vol. 170, no. 2, pp. 1452-1468, 2005.

[14] F. Yin and Y. Li, "Positive periodic solutions of a single species model with feedback regulation and distributed time delay," Applied Mathematics and Computation, vol. 153, no. 2, pp. 475-484, 2004.

[15] F. Chen, "Permanence in nonautonomous multi-species predator-prey system with feedback controls," Applied Mathematics and Computation, vol. 173, no. 2, pp. 694-709, 2006.

[16] F. Chen, "Permanence for the discrete mutualism model with time delays," Mathematical and Computer Modelling, vol. 47, no. 3-4, pp. 431-435, 2008.

[17] X. Liao, S. Zhou, and Y. Chen, "Permanence and global stability in a discrete $n$-species competition system with feedback controls," Nonlinear Analysis: Real World Applications, vol. 9, no. 4, pp. 1661$1671,2008$.

[18] S. Lu, "On the existence of positive periodic solutions to a Lotka Volterra cooperative population model with multiple delays," Nonlinear Analysis: Theory, Methods E Applications, vol. 68, no. 6, pp. 1746-1753, 2008.

[19] Z. Liu, R. Tan, Y. Chen, and L. Chen, “On the stable periodic solutions of a delayed two-species model of facultative mutualism," Applied Mathematics and Computation, vol. 196, no. 1, pp. 105-117, 2008.

[20] Z. Liu and L. Chen, "Periodic solutions of a discrete time nonautonomous two-species mutualistic system with delays," Advances in Complex Systems, vol. 9, no. 1-2, pp. 87-98, 2006.

[21] Y. Li and H. Zhang, "Existence of periodic solutions for a periodic mutualism model on time scales," Journal of Mathematical Analysis and Applications, vol. 343, no. 2, pp. 818-825, 2008.

[22] H. Wu, Y. Xia, and M. Lin, "Existence of positive periodic solution of mutualism system with several delays," Chaos, Solitons E Fractals, vol. 36, no. 2, pp. 487-493, 2008.

[23] Y. Xia, J. Cao, and S. S. Cheng, "Periodic solutions for a Lotka-Volterra mutualism system with several delays," Applied Mathematical Modelling, vol. 31, no. 9, pp. 1960-1969, 2007. 


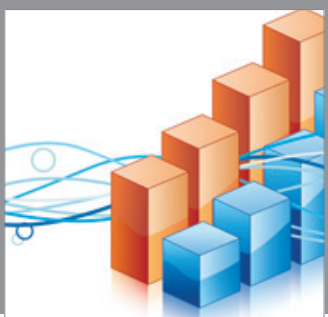

Advances in

Operations Research

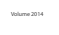

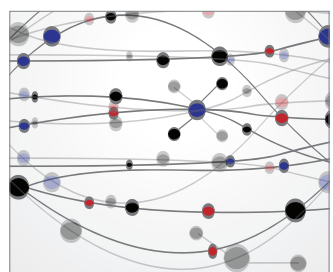

\section{The Scientific} World Journal
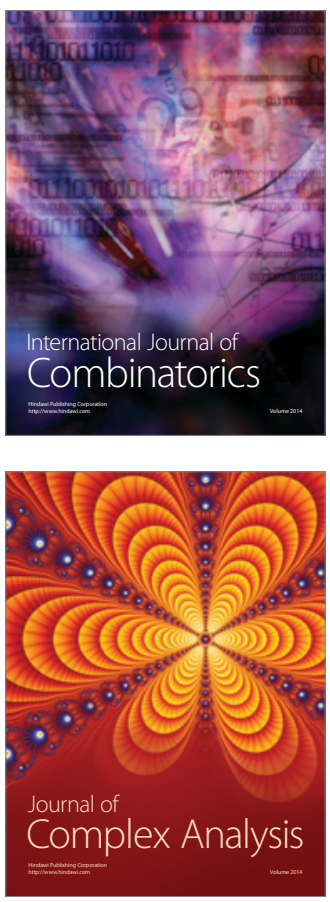

International Journal of

Mathematics and

Mathematical

Sciences
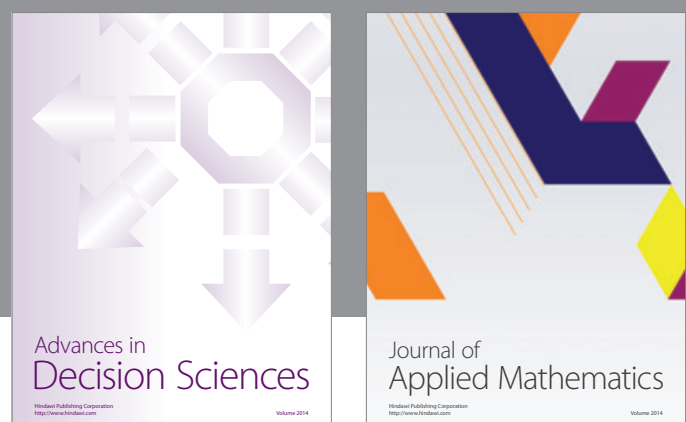

Journal of

Applied Mathematics
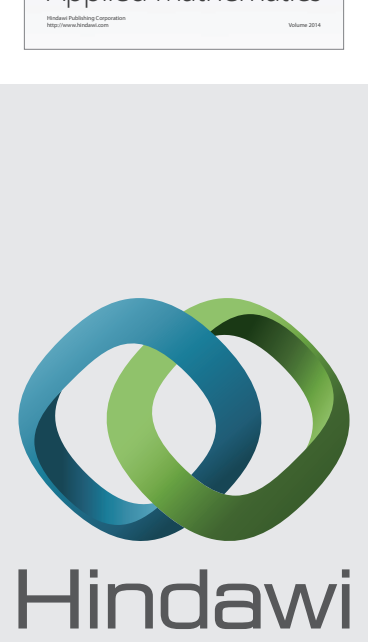

Submit your manuscripts at http://www.hindawi.com
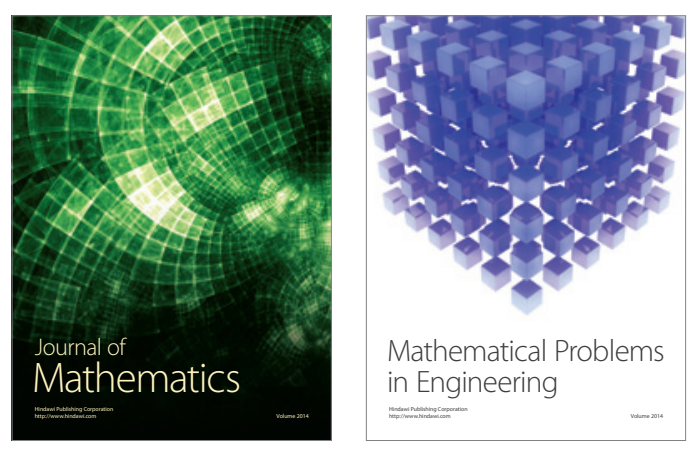

Mathematical Problems in Engineering
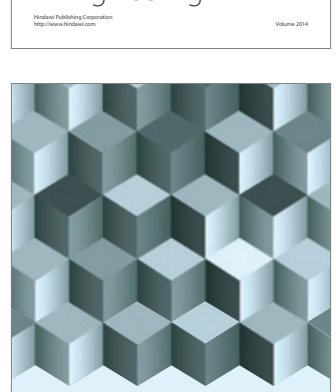

Journal of

Function Spaces
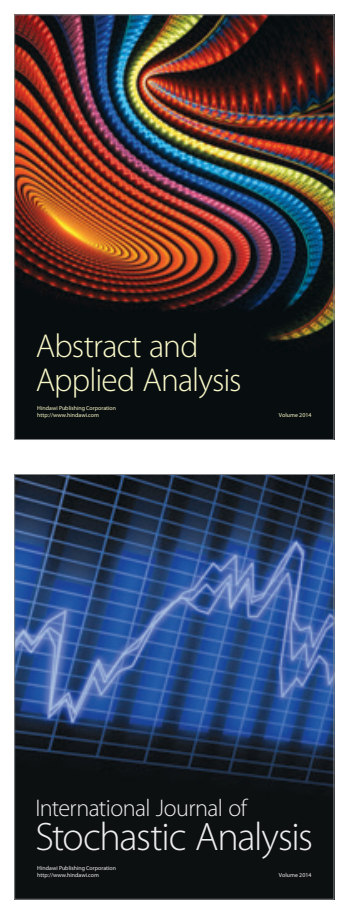

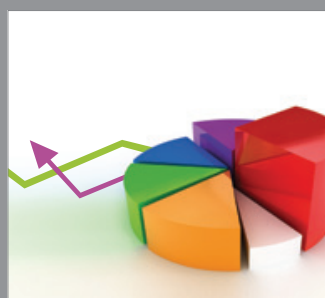

ournal of

Probability and Statistics

Promensencen
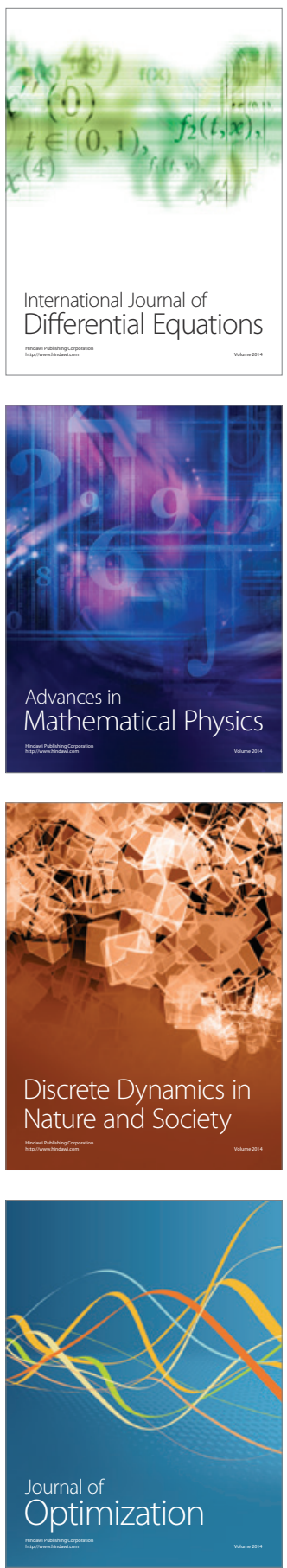